\title{
Quality Control Chart Design Under Jidoka
}

\author{
Emre Berk, ${ }^{1}$ Ayhan Özgür Toy \\ ${ }^{1}$ Department of Business Administration, Bilkent University, Ankara, Turkey \\ ${ }^{2}$ Department of Industrial Engineering, Turkish Naval Academy, Istanbul, Turkey
}

Received 23 November 2006; revised 20 January 2009; accepted 4 February 2009

DOI 10.1002/nav.20357

Published online 11 May 2009 in Wiley InterScience (www.interscience.wiley.com).

\begin{abstract}
We consider design of control charts in the presence of machine stoppages that are exogenously imposed (as under jidoka practices). Each stoppage creates an opportunity for inspection/repair at reduced cost. We first model a single machine facing opportunities arriving according to a Poisson process, develop the expressions for its operating characteristics and construct the optimization problem for economic design of a control chart. We, then, consider the multiple machine setting where individual machine stoppages may create inspection/repair opportunities for other machines. We develop exact expressions for the cases when all machines are either opportunity-takers or not. On the basis of an approximation for the all-taker case, we then propose an approximate model for the mixed case. In a numerical study, we examine the opportunity taking behavior of machines in both single and multiple machine settings and the impact of such practices on the design of an $\bar{X}-\mathrm{Q} \mathrm{C}$ chart. Our findings indicate that incorporating inspection/repair opportunities into QC chart design may provide considerable cost savings. (C) 2009 Wiley Periodicals, Inc. Naval Research Logistics 56: 465-477, 2009
\end{abstract}

Keywords: statistical process control; control chart design; Jidoka process control; opportunistic inspection

\section{INTRODUCTION}

In this article, we consider design of a quality control chart for a process facing exogenous stoppages, which act as opportunities for inspection/repair at reduced cost. Any realistic industrial process consists of various components/machines which are operated under a maintenance and/or quality control policy and need to be stopped for inspection/repair. In some cases, a machine's stoppage may result in a systemwide stoppage because of technical reasons-for example, a turbine stoppage shuts down the entire power plant. In other cases, it may be a managerial policy to force a systemwide stoppage when a single machine is stopped. For example, under the practice of jidoka (also called autonomation), whenever a machine is stopped due to whatever reason, the entire production line is stopped to prevent any value from being added on to any defective units $[14,20,24]$. Herein we do not differentiate between technical or managerial reasons for system stoppages but, for ease of exposition, refer to the setting with exogenous stoppages as a jidoka setting. In this setting, we propose that the process is operated under

Additional Supporting Information may be found in the online version of this article.

Correspondence to: E. Berk (eberk@bilkent.edu.tr) the jidoka process control (JPC), which is a combination of the conventional use of control charts and randomly occurring system stoppages for inspection/repair decisions. Each system stoppage requires the machine to be stopped and, as such, may create an opportunity for inspection/repair of the machine at a reduced downtime cost. Such opportunities have been considered for determining new policies in the maintenance literature after the seminal work by Dekker and Dijkstra [6]; but they have not received any attention in the vast quality control literature.

Quality control charts have been developed from three perspectives: a purely statistical approach where the power of the test for detecting an assignable cause and value for Type I error are set to their predetermined values $[15,19]$, a purely economic approach where the objective is minimization of the expected total costs of sampling, poor quality and downtime $[7,16,18]$, and a mixed approach called semi-economic design [22]. For comprehensive surveys and reviews of this extensive literature, we refer the reader to Refs. 10,13, 17,23, and 26. In this article, we focus on the economic design of control charts but the operating characteristics developed herein can be used for other types of control chart design, as well.

We begin our analysis with modeling a single machine facing exogenous opportunities, and then using this as a 
building block, we propose models for the multiple machine setting where some of the machines may be opportunitytakers and some not. Through a numerical study, we show that significant savings may be achieved by using the models developed herein versus the classical models in the presence of opportunistic inspections.

The rest of the article is organized as follows. In Section 2 , we present the basic assumptions for the single machine setting. In Section 3, we develop the single machine model. In Section 4, we provide a numerical study on the single machine. In Section 5, we generalize to the multiple machine setting and provide exact and approximate models for this setting. Section 6 discusses our numerical study for the multiple machine setting. Finally, we conclude in Section 7.

\section{SINGLE MACHINE SETTING: BASIC ASSUMPTIONS}

We consider a production process characterized by a single in-control status and a single out-of-control status; we use the terms process and machine interchangeably. We assume that there is a single assignable cause. Initially and after each intervention, the process is in the in-control status, producing items of acceptable quality when operational. After some time in production, the process shifts to the out-of-control status. Occurrence of the single assignable cause constitutes the shift in the process parameters. From then on, items of unacceptable quality are produced until an intervention occurs. There is a single quality characteristic, $X$, by which the process is evaluated and controlled; the parameters of the distribution of $X$ also change with the in-control and out-of-control status. The assignable cause is assumed to be nonobservable so that inference about the status of the process can only be drawn indirectly through observation of a sample statistic of $X$ computed at every sampling interval $h$ based on a sample of size $y$. The elapsed time until the process shift is distributed exponentially with mean $1 / \lambda$ (see Refs. $2-4,7,9,12,16$ for similar assumption and Refs. 5 and 8 for its empirical support).

The production process is not self-correcting. Defective items are eventually discarded at some cost. Although considered in isolation, the production process at hand is assumed to constitute a part of a bigger system operated under the principles of jidoka so that it undergoes forced (system-wide) shutdowns originating from the rest of the system. The exogenous shutdowns with a fixed duration of $L_{O}$ are assumed to arrive according to a Poisson process with mean $\mu$.

Before we proceed with the proposed quality control policy, a few remarks are in order regarding the memoryless nature of the exogenously forced shutdowns. In a complex production system where jidoka is employed, there will also be some system-wide forced shutdowns, which originate from the other machines in the system and arise from the alarms signalled on those machines. When there are a large number of machines in the system and/or when the sampling instances are different, because of, for example, different reliability and cost parameters of the machines, it will appear to a particular machine that the shutdowns come randomly. An assembly line typically consists of tens of workstations working in tandem. When autonomation is employed on such a line, the population from which the stoppages come is very large. Assuming that the sampling intervals are different, as they would be in general for nonidentical machines as workstations, it is reasonable to assume that there is a positive probability that a shutdown signal may be issued in a small time increment. Given the exponential nature of shift occurrences and the large number of machines involved in the population, it is again reasonable to assume that the stoppage probability over a time increment is stationary.

In this setting, we propose that the process is operated under what we call the jidoka process control (JPC), which is a combined usage of the control charts and randomly occurring opportunity-based inspections as follows. A sample of size $y$ is taken from the process at prespecified intervals of length $h$. The sample unit(s) are analyzed and measured. The sample statistic of the quality characteristic is computed and checked against prespecified control limits defined through the control parameters $k$ and $y$ as $\mu_{0}+k \sigma / \sqrt{y}$ and $\mu_{0}-k \sigma / \sqrt{y}$ in an $\bar{X}$ control chart for $X$ which is continuous and $X \sim N\left(\mu_{0}, \sigma\right)$ when in the in-control status and $X \sim N\left(\mu_{0} \pm \delta \sigma, \sigma\right)$ when in the out-of-control status. If it is outside the control limits, an inspection of the process or a search for the assignable cause is conducted. If the process is indeed in the out-of-control status, the signal is said to result in a true alarm followed by a complete restoration of the process to the in-control status; otherwise, the signal results in a false alarm which requires no adjustment or restoration. A sample results in a false alarm with probability $\alpha$ (Type I error) and a true alarm with probability $(1-\beta)$ (complement of Type II error). Clearly, $\alpha$ and $\beta$ are related to $y$ and $k$ as $\alpha=2 \Phi(-k) ; \beta=\Phi(k-\delta \sqrt{y})-\Phi(-k-\delta \sqrt{y})$ for a normal variate $X$. So far, it is supposed that the process stops itself; this is the standard statistical process control (SPC) scheme. Under the proposed JPC policy, the process at hand also acts as an opportunity-taker. That is, if the process faces an exogenous shutdown, its operator uses this stoppage as an opportunity to carry out an inspection of the process although no signals have been received from the control chart to initiate one. On inspection, if the process is found to be in the out-of-control status, the opportunity is said to be a true opportunity which is followed by a complete restoration of the process to the in-control status; otherwise, the opportunity is a false opportunity which requires no adjustment. Thus, under JPC, the process stops either by itself via an alarm arising from the inferring procedure or by an exogenous 
opportunity generated by a system-wide shutdown. Assuming perfect repair/restoration after each stoppage, the process restarts in the in-control status.

The instances at which the process restarts are regeneration points, since, at each restart, the process is in the in-control status, and occurrences of shifts and opportunities are memoryless processes. Therefore, we can define a regenerative cycle as the time between two consecutive process restarts. We identify four cycle classes $s \in\{T, F, O T, O F\}$ where, $T$ denotes the class of cycles in which a true alarm triggers the process stoppage and the process is in the out-of-control status at the time of stoppage; $F$ denotes the class of cycles in which a false alarm triggers the process stoppage and the process is in the in-control status at the time of stoppage; $O T$ denotes the class of cycles in which a true opportunity triggers the process stoppage and the process is in the outof-control status at the time of stoppage; and, finally, $O F$ denotes the class of cycles in which a false opportunity triggers the process stoppage and the process is in the in-control status at the time of stoppage.

We assume that the entire process of analysis for a sample takes negligible time, whereas, both the search for the assignable cause and the possible restoration of the process necessitate the stoppage of a machine and take non-negligible time. The durations of search and restoration may depend on the status of the process.

We consider the following categories of costs: (i) Cost of sampling and testing, which is given by $u+b y$ for a sample of size $y$. (ii) The cost associated with production of defective items expressed in terms of the cost of operating in the out-ofcontrol status taken as $a$ per time unit, due to, for example, substandard outputs. Finally, (iii) the costs associated with investigation and correction of the assignable cause of variation, which consist of out-of-pocket repair or replacement costs due to, for example, scrapped components and destructive inspection, and opportunity costs of foregone profit due to downtime of the machine. For cycle class $s, R_{s}$ is the outof-pocket component and the opportunity cost component is computed as $\pi L_{s}$, where $\pi$ is the foregone profit per unit of time and $L_{s}$ is the downtime of the system attributed to the machine. For brevity, we shall refer to the activities of inspection, investigation, and correction of the assignable cause, if any, as inspection/repair.

The objective is to determine the control parameters $(y, k, h)$ which minimize the expected cost per operating time, $E[T C]$, referred to as the expected cost rate. In our construction, we work with the operating time rather than the chronological time. That is, we consider only the time segments during which the process/machine is "working/producing". We chose to work with cost per operating time for the simple reason that it enables us to use this cost rate as a direct proxy for production cost per unit of product. If the production rate is constant and any defective item coming out of the process is either reworked offline or discarded completely, then, $E[T C]$ will indeed be the expected cost per unit produced. We believe that this is a cost measure which is directly usable for cost accounting and pricing purposes and, hence, more meaningful for product managers. From the Renewal Reward Theorem (p.318 [21]), we can write $E[T C]$ as the ratio of the expected cycle cost, $E[C C]$ to the expected operating time in a cycle, $E[\tau]$.

\section{SINGLE MACHINE: OPERATING CHARACTERISTICS}

In this section, we derive the expressions for the operating characteristics of the single machine system and construct the objective function.

A cycle can be fully described by the quintuple $\left(S, N_{1}, N_{2}, X, Z\right)$ in which, $S$ refers to the cycle class; $X$, the time elapsed since the beginning of the cycle until the machine is stopped or a process shift occurs, whichever occurs first; $Z$, the time elapsed since the beginning of the cycle until the machine is stopped or an opportunity arrives, whichever occurs first; $N_{1}$, the number of samples taken before the shift has occurred; and, $N_{2}$, the number of samples taken after the shift in that cycle. Each class $S$ will have only certain permissible values for $X$ and $Z$ (and, thereby, for $N_{1}$ and $\left.N_{2}\right)$, as we shall discuss shortly. Let $\left(s, n_{1}, n_{2}, x, z\right)$ be a particular realization of this quintuple with the set of its permissible values denoted by $\Omega=\Omega(T) \cup \Omega(F) \cup$ $\Omega(O T) \cup \Omega(O F)$, where $\Omega(T)=\left\{\left(s, n_{1}, n_{2}, x, z\right): s=\right.$ $\left.T, n_{1} \geq 0, n_{2} \geq 1, n_{1} h<x<\left(n_{1}+1\right) h, z=\left(n_{1}+n_{2}\right) h\right\}$, $\Omega(F)=\left\{\left(s, n_{1}, n_{2}, x, z\right): s=F, n_{1} \geq 1, n_{2}=0, x=\right.$ $\left.z=\left(n_{1}+n_{2}\right) h\right\}, \Omega(O T)=\left\{\left\{\left(s, n_{1}, n_{2}, x, z\right): s=O T\right.\right.$, $\left.n_{1} \geq 0, n_{2}=0, n_{1} h<x<z<\left(n_{1}+1\right) h\right\} \cup\left\{\left(s, n_{1}\right.\right.$, $\left.n_{2}, x, z\right): s=O T, n_{1} \geq 0, n_{2}>0, n_{1} h<x<\left(n_{1}+1\right) h$, $\left.\left.\left(n_{1}+n_{2}\right) h<z<\left(n_{1}+n_{2}+1\right) h\right\}\right\}, \Omega(O F)=\left\{\left(s, n_{1}\right.\right.$, $\left.n_{2}, x, z\right): s=O F, n_{1} \geq 0, n_{2}=0, n_{1} h<x=z<$ $\left.\left(n_{1}+1\right) h\right\}$. Also let $f_{\left(S, N_{1}, N_{2}, X, Z\right)}\left(s, n_{1}, n_{2}, x, z\right)$ denote the joint probability function of the cycle described by the quintuple, and $\tau\left(s, n_{1}, n_{2}, x, z\right)$ denote the operating time within the corresponding cycle. Clearly, for $\left(s, n_{1}, n_{2}, x, z\right) \in \Omega$, $\tau\left(s, n_{1}, n_{2}, x, z\right)=z$ and

$$
\begin{aligned}
& f\left(s, n_{1}, n_{2}, x, z\right) \\
& = \begin{cases}(1-\alpha)^{n_{1}} \beta^{\left(n_{2}-1\right)} & \text { for }\left(s, n_{1}, n_{2}, x, z\right) \in \Omega(T) \\
(1-\beta) e^{-\mu z} \lambda e^{-\lambda x} & \text { for }\left(s, n_{1}, n_{2}, x, z\right) \in \Omega(F) \\
(1-\alpha)^{\left(n_{1}-1\right)} \alpha e^{-\mu z} e^{-\lambda x} & \text { for }\left(s, n_{1}, n_{2}, x, z\right) \in \Omega(O T) \\
(1-\alpha)^{n_{1}} \beta^{n_{2}} \mu e^{-\mu z} \lambda e^{-\lambda x} & \text { for }\left(s, n_{1}, n_{2}, x, z\right) \in \Omega(O F) \\
\mu e^{-\mu z} e^{-\lambda x}(1-\alpha)^{n_{1}} & \text { otherwise } \\
0 & \end{cases}
\end{aligned}
$$


A cycle with a realization $\left(s, n_{1}, n_{2}, x, z\right)$ incurs sampling costs for the $\left(n_{1}+n_{2}\right)$ many samples taken, the cost associated with operating in the out-of-control status and the costs for investigation and correction of the process. Hence, the cost incurred within a cycle excluding the downtime cost is

$$
\begin{array}{r}
C\left(s, n_{1}, n_{2}, x, z\right)=\left(n_{1}+n_{2}\right)(u+b y)+a[z-x]+R_{s} \\
\text { for }\left(s, n_{1}, n_{2}, x, z\right) \in \Omega(s) .
\end{array}
$$

Let $P_{s}(\mu)$ be the probability that the machine is in status $s$ at time of stoppage for a given opportunity rate $\mu ; P_{s}(\mu)=$ $\sum_{\left\{n_{1}, n_{2}\right\} \in \Omega(s)} \int_{\{x, z\} \in \Omega(s)} f\left(s, n_{1}, n_{2}, x, z\right) d x d z$. Then, the conditional expected length of operating time given the cycle is of class $s$ is

$$
\begin{array}{r}
E[\tau \mid S=s]=\sum_{\left\{n_{1}, n_{2}\right\} \in \Omega(s)} \int_{\{x, z\} \in \Omega(s)} \tau\left(s, n_{1}, n_{2}, x, z\right) \\
\cdot \frac{f\left(s, n_{1}, n_{2}, x, z\right)}{P_{s}(\mu)} d x d z
\end{array}
$$

Similarly, the conditional expected total cycle cost given the cycle is of class $s$ is

$$
\begin{array}{r}
E[C C \mid S=s] \\
=\sum_{\left\{n_{1}, n_{2}\right\} \in \Omega(s)} \int_{\{x, z\} \in \Omega(s)}\left[C\left(s, n_{1}, n_{2}, x, z\right)+\pi L_{s}\right] . \\
\frac{f\left(s, n_{1}, n_{2}, x, z\right)}{P_{s}(\mu)} d x d z
\end{array}
$$

(The individual expressions of $P_{s}(\mu), E[\tau \mid S=s]$ and $E[C C \mid S=s]$ for each $s$ value are provided in the Online Supplement.) A schematic representation of the evolution of a production process under JPC is depicted in Fig. 1.

The individual activities involved in inspecting the machine to identify the assignable cause of variation and restoring the process to its in-control status are prespecified, and, hence, their actual duration do not depend on whether the machine was stopped by itself or an opportunity. However, the effective durations of those activities will be different for each case. When the machine is stopped by an alarm, the effective durations of the search and possible restoration activities are their actual durations. Let $L_{s}$ denote the effective search and restoration time for a cycle in class $s$. Then, $L_{s}$ takes on values $L_{T}, L_{F}, \max \left[L_{T}, L_{O}\right]$ and $\max \left[L_{F}, L_{O}\right]$ for $s=T, F, O T$ and $O F$, respectively. The effective out-ofpocket repair costs are such that $R_{O T}=R_{T}$ and $R_{O F}=R_{F}$ because the activities are prespecified.

The optimization problem is formally stated as follows.

$$
\operatorname{Minimize}_{y, h, k>0} E[T C]=\frac{E[C C]}{E[\tau]}
$$

Note that the cost is per operating time excluding the downtime. The expected length of the operating time within a cycle, $E[\tau]$ is given by:

$$
E[\tau]=\sum_{s \in\{T, F, O T, O F\}} E[\tau \mid S=s] \cdot P_{s}(\mu)
$$

Similarly, the expected cycle cost, $E[C C]$ is given by

$$
E[C C]=\sum_{s \in\{T, F, O T, O F\}} E[C C \mid S=s] \cdot P_{s}(\mu)
$$

REMARK 1: The expressions for the operating characteristics reduce to the classical ones in the absence of exogenous opportunities as $\lim _{\mu \rightarrow 0}[7,9]$.

REMARK 2: So far, we have assumed that the opportunities are of a single kind. However, in reality, there may be different sources of opportunities with different durations. In the presence of opportunities of such random durations with a known probability distribution, the analysis above holds with a slight modification. The opportunistic inspection/repair times are now expected values denoted by $\bar{L}_{O T}$ and $\bar{L}_{O F}$ with the expectation taken over the random variable $L_{O}$. The self-triggered inspection/repair times may also be represented as $\bar{L}_{T}\left(=L_{T}\right)$ and $\bar{L}_{F}\left(=L_{F}\right)$ for notational convenience.

\section{SINGLE MACHINE: NUMERICAL STUDY}

In our numerical study for the single machine setting, we examined (i) the sensitivity of the optimal values of the control policy parameters and the expected cost rate w.r.t. the system and cost parameters, (ii) the advantages of using the optimal control policy parameters as computed with the model herein versus using the classical model parameters, in the presence of exogenous opportunities of inspection/repair, and (iii) whether or not JPC is beneficial in a particular setting.

The cost rate function of the classical model, which is a special case of the model herein, is known to be unimodal jointly in the policy triplet $(y, k, h)$ [11]. We have not observed an instance that violates the unimodality of the objective function under JPC in an extensive preliminary numerical study; but, we have not been able to prove it analytically. We employed golden section search ([1], p. 270) for determining the optimal values of $k$ and $h$ in the finite intervals $[0.001,50]$ and $[0.001,50]$, and an exhaustive search for $y$ over $[1,50]$ with an increment size of one. For the experimental design, we used the system parameter set given in Table 1 with the opportunity arrival rate taken as multiples of the shift rate, $\mu \in\{0,0.5 \lambda, \lambda, 1.5 \lambda, 2 \lambda, 5 \lambda, 10 \lambda\}$. Overall, we generated 5103 different experiment instances for our numerical study. (See [25] for algorithm details.) 

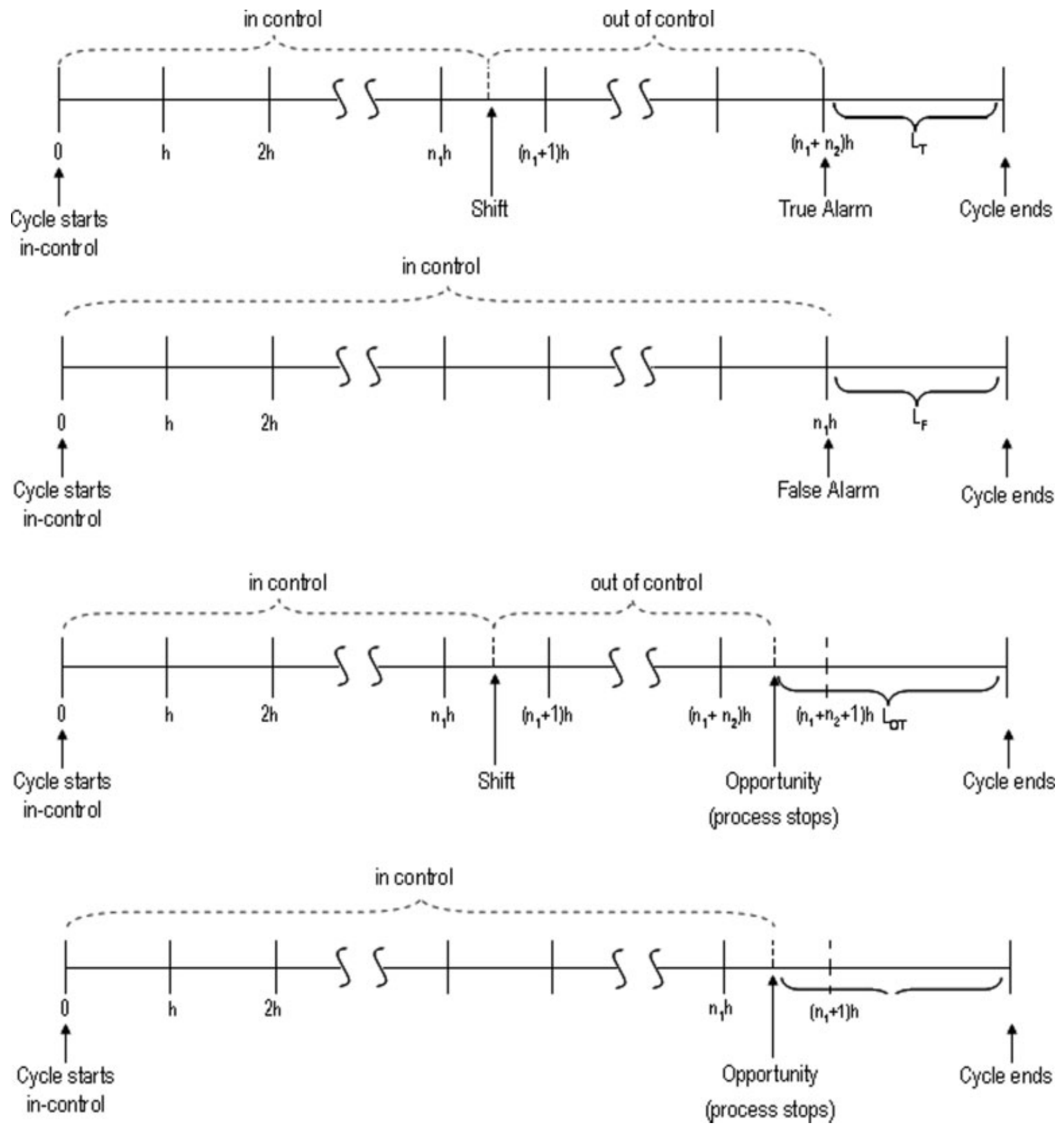

Figure 1. Cycle types. (a) True cycle, (b) False cycle, (c) Opportunity True cycle, (d) Opportunity False cycle.

\subsection{Sensitivity Analysis}

The behavior of the optimal expected cost rate, $E\left[T C^{*}\right]$ and the optimal control policy triplet $\left(y^{*}, k^{*}, h^{*}\right)$ w.r.t. the cost parameters $(u, b, a)$ are similar to those observed in the classical case. The effect of $L_{O}$ depends on $L_{T}$ and $L_{F}$. The optimal cost rate is decreasing in the opportunity duration. The sample size and control limit coefficient are insensitive to $L_{O}$. For small values of $L_{T}$ relative to $L_{F}, h^{*}$ is decreasing in $L_{O}$; but for large $L_{T}$ relative to $L_{F}$, it shows an increasing trend. For example, for $L_{T}=0.1$ and $L_{F}=0.5$, $\left.h^{*}\right|_{L_{o}=0.1}>\left.h^{*}\right|_{L_{o}=0.25}>\left.h^{*}\right|_{L_{o}=0.5}$; for $L_{T}=0.25$ and $L_{F}=$ $0.5,\left.h^{*}\right|_{L_{o}=0.1} \leq\left. h^{*}\right|_{L_{o}=0.25}>\left.h^{*}\right|_{L_{o}=0.5}$; and, for $L_{T}=0.5$ and $L_{F}=0.5$, we have $\left.h^{*}\right|_{L_{o}=0.1} \leq\left. h^{*}\right|_{L_{o}=0.25}<\left.h^{*}\right|_{L_{o}=0.5}$. A summary of the sensitivity study results are presented in Table 2. 
Table 1. The parameter set for the single machine numerical study.

\begin{tabular}{cccc}
\hline Parameter & \multicolumn{3}{c}{ Values } \\
\hline$\lambda$ & & 0.05 & \\
$\pi$ & 50 & 500 & 250 \\
$a$ & 0.1 & 100 & 1 \\
$b$ & 0 & 0.2 & 10 \\
$u$ & 0.1 & 0.25 & 0.5 \\
$L_{F}$ & 0.1 & 0.25 & 0.5 \\
$L_{T}$ & 0.1 & 0.25 & 0.5 \\
$L_{o}$ & & 0 & \\
$R_{F}$ & & 0 & \\
$R_{T}$ & & & \\
\hline
\end{tabular}

\subsection{Impact of Opportunity Arrival Rate, $\mu$}

We observe that changes in the optimal cost rate, $E\left[T C^{*}\right]$ w.r.t. the opportunity arrival rate depend solely on the relationship between $L_{O}$ and $L_{F}$. For $L_{O} \geq L_{F}$, regardless of $L_{F}$ and $L_{T}, E\left[T C^{*}\right]$ is decreasing in $\mu$; otherwise, it is increasing.

To explain the rationale behind this observation, first consider the case $\left(L_{O} \geq L_{F}\right)$ and $\left(L_{O} \geq L_{T}\right)$. Recall that, when the system stoppage is triggered by an opportunity, the machine incurs lost profit cost only for the additional time it delays the system restart, i.e. $L_{O T}\left(=\left[L_{T}-L_{O}\right]^{+}\right)$or $L_{O F}\left(=\left[L_{F}-L_{O}\right]^{+}\right)$. In case of a stoppage by an opportunity, the machine will be restored to the in-control status free of charge. More frequent opportunities are always beneficial in order to keep the machine in the in-control status and to provide savings in cost of operating in out-of-control status and cost of inspection and repair. Thereby, the overall cost rate decreases w.r.t. $\mu$. Next, consider the case $\left(L_{O} \geq L_{F}\right)$ and $\left(L_{O}<L_{T}\right)$. A similar argumentation applies: Opportunities arriving when the machine is in the in-control status can be taken at no cost; although $L_{O}<L_{T}$, more frequent opportunities contribute to the early detection of the out-of-control status with less cost. Hence, more frequent opportunities decreases the cost rate. We see that the decrease in cost rate gets steeper as $\left(L_{O}-L_{T}\right)$ increases. Finally, consider the case $\left(L_{O}<L_{F}\right)$ with either $\left(L_{O}<L_{T}\right)$ or $\left(L_{O}>L_{T}\right)$. In this case, stoppages due to opportunities are more costly, since the restoration time takes longer than the opportunistic inspection/restoration duration if the system is in the incontrol status at the stoppage instant. When the opportunity rate increases, it is more likely that the process will be in the in-control status when. Hence, the overall expected cost rate increases as the opportunity rate increases.

Our numerical results indicate that $k^{*}$ and $y^{*}$ are insensitive to the opportunity rate $\mu$. However, the sampling interval $h^{*}$ increases as the opportunity rate increases. When there are more frequent exogenous stoppages for inspection/repair, the process status can be assessed without inference from sampling. Therefore, sampling less frequently yields a lower sampling cost, resulting in a lower total cost rate.

\subsection{Advantages of JPC}

Next, we study the benefits of determining the optimal values of the control policy parameters as modeled herein versus using the classical SPC parameter values. The improvement achieved through the optimal determination of the policy parameters is given by

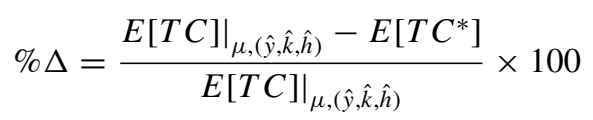

where $\left.E[T C]\right|_{\mu,(y, k, h)}$ denotes the expected cost rate evaluated with $(y, k, h)$ in the presence of exogenous opportunities with rate $\mu$ and $(\hat{y}, \hat{k}, \hat{h})=\left.\arg \min _{(y, k, h)} E[T C]\right|_{\mu=0}$ denotes the global minimizer values in the absence of opportunities $(\mu=0)$.

As expected, the percentage improvement is increasing in both the sampling costs, $u$ and $b$; and, it is decreasing in $a$. The effects lessen as $\mu$ increases.

Introduction of opportunistic inspections decreases reliance of the system on statistical inferencing; one would expect that the sampling scheme would get looser - longer sampling intervals, larger control limit coefficients - as the opportunities either increase in frequency or in durations. Hence, we would expect that, for moderate values of opportunity arrivals and durations, the percentage improvements achieved via modeling opportunistic inspections will increase as either $L_{O}$ or $\mu$ increases; but, that, with further increase in $L_{O}$ or $\mu, \% \Delta$ will start decreasing since the sampling scheme becomes less important in inferencing and stoppages would be caused mostly by exogenous opportunities. Our findings are consistent with this intuition.

The behavior of $\% \Delta$ w.r.t. $L_{T}$ depends only on the effective restoration time $L_{O T}$. The percentage improvement is increasing in $L_{T}$ as long as $L_{O T}$ is nonincreasing; otherwise, it is decreasing.

As the false restoration time, $L_{F}$ increases, the cost rate becomes more and more sensitive to false alarms. Hence,

Table 2. Summary of the sensitivity of optimal control parameter values and the expected cost rate w.r.t. system parameters; increase $(\uparrow)$, no change $(\longleftrightarrow)$ and decrease $(\downarrow)$.

\begin{tabular}{ccccc}
\hline & $y^{*}$ & $k^{*}$ & $h^{*}$ & $E\left[T C^{*}\right]$ \\
\hline$u$ & $\uparrow$ & $\leftrightarrow$ & $\uparrow$ & $\uparrow$ \\
$b$ & $\downarrow$ & $\downarrow$ & $\uparrow$ & $\uparrow$ \\
$a$ & $\leftrightarrow$ & $\downarrow$ & $\downarrow$ & $\uparrow$ \\
$L_{O}$ & $\leftrightarrow$ & $\leftrightarrow$ & $\uparrow$ & $\downarrow$ \\
$L_{T}$ & $\leftrightarrow$ & $\leftrightarrow$ & $\uparrow$ & $\uparrow$ \\
$L_{F}$ & $\uparrow$ & $\uparrow$ & $\uparrow$ & $\uparrow$ \\
\hline
\end{tabular}


Table 3. Summary statistics of the percentage improvement of JPC over classical SPC.

\begin{tabular}{lllllllc}
\hline & $\mu$ & 0.025 & 0.05 & 0.075 & 0.1 & 0.25 & 0.5 \\
\hline$L_{o}<L_{F}$ & Min & 0 & 0 & 0 & 0.01 & 0.04 & 0.12 \\
& Max & 0.21 & 0.77 & 5.02 & 6.83 & 9.48 & 8.32 \\
& Mean & 0.0445 & 0.153 & 0.383 & 0.546 & 2.207 & 2.672 \\
& Median & 0.023 & 0.09 & 0.17 & 0.27 & 1.11 & 2.72 \\
$L_{o} \geq L_{F}$ & Min & 0 & 0 & 0 & 0 & 0.03 & 0.12 \\
& Max & 0.23 & 1.04 & 7.69 & 11.37 & 34.5 & 59.84 \\
& Mean & 0.026 & 0.109 & 0.289 & 0.560 & 4.361 & 11.983 \\
Overall & Median & 0.01 & 0.05 & 0.11 & 0.19 & 1.225 & 5.585 \\
& Min & 0 & 0 & 0 & 0 & 0.03 & 0.12 \\
& Max & 0.23 & 1.04 & 7.69 & 11.37 & 34.5 & 59.84 \\
& Mean & 0.032 & 0.124 & 0.319 & 0.558 & 3.603 & 8.844 \\
& Median & 0.015 & 0.06 & 0.129 & 0.217 & 1.21 & 3.95 \\
\hline
\end{tabular}

$\% \Delta$ is increasing in $L_{F}$, except for the cases where the system relies little on inferencing. For large values of $\mu, u$, and $b$, process control relies mostly not on inferencing through sampling but rather on inspections at exogenously induced stoppages; hence, the savings due to optimal determination of the control policy triplet diminish for these cases.

The percentage improvements achieved with the model herein monotonically increase as $L_{O}$ gets larger, except for the cases where false alarms are too costly so that selfstoppages are infrequent. For instance, for $L_{T}=0.1$ and $L_{F}=0.5, \% \Delta$ exhibits an almost convex behavior in $L_{O}$ for small to medium opportunity rates; but, for large $\mu$, it is increasing. As the cost of operating in the out-of-control status, $a$ increases, the effect gets more pronounced. As $L_{O}$ exceeds $L_{T}$ and $L_{F}$, the increase in $\% \Delta$ w.r.t. $L_{O}$ gets very small, as expected.

In Table 3 we provide the summary statistics of $\% \Delta$ for (i) the overall experimental set, (ii) those instances with $L_{O}<L_{F}$, and (iii) those instances with $L_{O} \geq L_{F}$, at different levels of $\mu$. The maximum saving was observed for the instance where $L_{T}=0.5, L_{F}=0.1, L_{O}=0.5, a=50$, $u=0, b=0.1$, and $\mu=0.5$. We conclude that savings can be significant if the control policy parameters are determined optimally under JPC.

\section{MULTIPLE MACHINE SETTING}

\subsection{Preliminaries}

In the single machine model, inspection opportunities were treated as exogenous. In this section, we develop the multiple machine model where such opportunities are generated within the system due to individual machine stoppages. Every time an alarm is raised and the system stoppage is triggered by a machine, it creates an inspection opportunity for the rest of the machines in the system. We envision the multiple machine setting as a production system where the machines operate in accordance with the jidoka philosophy. As illustrated in Section 4.2, it is not always beneficial for a particular machine to take the stoppage opportunities depending on system parameters; in a multiple machine setting, some machines may take the opportunities whereas the rest may not. We will designate a machine that utilizes the opportunities as an opportunity taker, and a machine that does not utilize the opportunities as an opportunity non-taker. In general, there are three possible partitionings of the machines in a system: (i) the all non-taker case where all of the machines are opportunity non-takers, (ii) the all taker case where all of the machines are opportunity takers, or (iii) the mixed case where some of the machines are opportunity takers and the rest are not. We discuss each case separately. For the two pure cases, we construct exact models. We also develop an approximate model for the all-taker case based on the single machine model with Poisson exogenous opportunities. Using this heuristic approach, we provide an approximate model for the mixed case.

Before we proceed, we briefly point out the fundamental differences between the single and multiple machine settings.

1. In the single machine model, we have assumed that opportunity arrival times follow an exponential distribution with a given rate $\mu$. But, in the multiple machine setting, (i) the opportunity arrival process is not necessarily Markovian and (ii) the machine partitioning, the status and reliability of all machines and their control policy parameters jointly determine the opportunity arrival rate experienced by each machine.

2. A system regeneration point in the single machine setting coincides with a machine restart instance, which follows every stoppage. When there are opportunity taker and opportunity nontaker machines together in the system, those opportunity nontaker machines which are in the out-of-control status at the stoppage instant will not be restored to their incontrol status. For an opportunity nontaker machine to be in the in-control status at a system restart, either it must be in the in-control status at the previous stoppage instance or the stoppage must have been triggered by itself. Thus, system restarts, by themselves, do not always correspond to system regeneration points in the multiple machine setting.

3. The cost computation of the multiple machine case also requires additional care. The sampling cost and the cost of operating in the out-of-control status are still incurred by individual machines; but, the idleness cost is incurred by the overall system, unlike in the single machine case. 
We introduce some common notation. $\mathcal{M}$ denotes the set of machines with cardinality $|\mathcal{M}|=m$. We retain the notation for the single machine case, and use the superscript ${ }^{(i)}$ to denote the parameters of a particular machine $i(\in \mathcal{M})$. For every machine $i$ in the system, a three-parameter quality control policy is employed. The set of opportunity taker machines is denoted by $\mathcal{M}_{T K}$, and the set of opportunity nontaker machines by $\mathcal{M}_{N T K}$. Clearly, $\mathcal{M} \equiv \mathcal{M}_{T K} \cup \mathcal{M}_{N T K}$ and $\mathcal{M}_{T K} \cap \mathcal{M}_{N T K} \equiv \emptyset$. The cardinalities of $\mathcal{M}_{T K}$ and $\mathcal{M}_{N T K}$ are $m_{T K}$ and $m_{N T K}$, respectively. Also $\mathcal{M}^{\prime} \equiv\left\{\mathcal{M}_{T K} \cup\{j\}\right\}$ denotes the set of machines that will be inspected at the system stoppage instance, triggered by machine $j$. The cardinality of $\mathcal{M}^{\prime}$ is $m^{\prime}$ (Note that $m^{\prime}=m_{T K}$ if $j \in \mathcal{M}_{T K}$ and $m^{\prime}=m_{T K}+1$ otherwise). We suppress the index $j$ of $\mathcal{M}^{\prime}$ for brevity as it will be clear from the context. Let $\delta^{(i)}$ be the binary variable whether machine $i$ is an opportunitytaker; $\delta^{(i)}=1$ iff $i \in \mathcal{M}_{T K}$, and 0 , otherwise. Given a set of machines $\mathcal{M}$, the objective is to determine: (i) the optimum control parameters, $y^{*(i)}, h^{*(i)}$ and $k^{*(i)}$ for each machine $i(\in \mathcal{M})$ and (ii) the optimum partitioning of the machines into the opportunity taker and opportunity nontaker sets, so that the long run expected cost per unit of operating time, $E[T C]$, is minimized. (As in the single machine model, we shall refer to $E[T C]$ as the expected cost rate, for brevity.) We formally state the optimization problem as follows.

$$
\min _{y^{(i)}, h^{(i)}, k^{(i)}, \delta^{(i)} \forall i \in \mathcal{M}} E[T C]
$$

Next, we develop the models for the three cases of machine partitionings.

\subsection{All Opportunity Nontaker Model}

All of the machines are opportunity nontakers, i.e., $\mathcal{M}_{\mathrm{TK}}=$ $\emptyset, m=m_{\mathrm{NTK}}$, and $\delta^{(i)}=0 \forall i$. In this case, machines are inspected and repaired only through self-stoppages. Define a cycle for machine $i\left(\in \mathcal{M}_{\mathrm{NTK}}\right)$ as the time between two consecutive self-stoppages of that machine. The machines may, then, be viewed as going through regenerative cycles independently. Invoking the Renewal Reward Theorem, the expected cost per operating time for the $m$ machine system is given by the sum of expected cost rates incurred by individual machines facing no opportunities:

$$
E[T C]=\lim _{t \rightarrow \infty} \frac{\sum_{i \in M} R_{i}(t)}{t}=\sum_{i \in M} \frac{\left.E\left[C C^{(i)}\right]\right|_{\mu^{(i)}=0}}{\left.E\left[\tau^{(i)}\right]\right|_{\mu^{(i)}=0}}
$$

where $t$ denotes the cumulative operating time, $R_{i}(t)$ denotes the cumulative cost incurred by machine $i$ up to $t, E\left[C C^{(i)}\right]$ and $E\left[\tau^{(i)}\right]$ are as given in Eqs. (7) and (6). As shutdowns are not utilized for inspecting other machines, the downtime cost is charged only to the machine that stops the system. (Note that the probability of simultaneous stoppage of more than one machine would be very small; hence, such an occurrence can be safely neglected.) The cost per unit of downtime is the same for all machines $\left(\pi^{(i)}=\pi \forall i\right)$.

\subsection{All Opportunity Taker Model}

All of the machines are opportunity takers, i.e., $\mathcal{M}_{\mathrm{NTK}}=$ $\emptyset, m_{\mathrm{TK}}=m$ and $\delta^{(i)}=1 \forall i$. In this case, at each system restart, each machine $i\left(\in \mathcal{M}_{\mathrm{TK}}\right)$ is in the in-control status and the time to its first sampling instance is exactly $h^{(i)}$. Therefore, each system restart is a regeneration point for all of the machines and, hence, for the overall system. Suppose the following scenario. Machine $j$ signals an alarm which causes a self-stoppage and an exogenous stoppage for the rest of the machines. At the time of stoppage, $s^{(j)} \in\{T, F\}$ and $s^{(i)} \in\{O T, O F\} \forall i \in \mathcal{M}_{\mathrm{TK}} \backslash\{j\}$ depending on whether or not a process shift has occurred for a particular machine. Let $L_{\max }(j, \mathbf{s})$ be the maximum inspection/repair time of the overall system, and $i_{\max }(j, \mathbf{s})$ be the index of that machine with the maximum inspection/repair time when the system stoppage is triggered by machine $j$ and the status of the machines in $\mathcal{M}^{\prime}$ are given by the vector $\mathbf{s}$. Then, $L_{\max }(j, \mathbf{s})=$ $\max _{k \in \mathcal{M}} /\left\{L_{T}^{(k)} I_{s^{(k)} \in\{T, O T\}}, L_{F}^{(k)} I_{s^{(k)} \in\{F, O F\}}\right\}$, and $i_{\max }(j, \mathbf{s})=$ $\arg \max _{k \in \mathcal{M}}\left\{L_{T}^{(k)} I_{s^{(k)} \in\{T, O T\}}, L_{F}^{(k)} I_{s^{(k)} \in\{F, O F\}}\right\}$. The time and cost of inspection/repair for each machine at a system stoppage are computed as follows. Machine $j$ will incur the portion of the downtime cost corresponding to its own inspection/repair time. Machine $i_{\max }(j, \mathbf{s})$ will experience an opportunity duration equal to the inspection/repair time of machine $j$. If $i_{\max }(j, \mathbf{s})=j$, there will be no additional delay for the system; otherwise, there will be a positive delay and machine $i_{\max }$ will incur the additional downtime cost. The rest of the machines will experience opportunistic inspection/repair times of zero duration, and incur zero idleness costs. Thus, the opportunity duration $L_{O}^{(i)}(j, \mathbf{s})=I_{(i \neq j)}\left[I_{\left(s^{(j)}=T\right)} L_{T}^{(j)}+\right.$ $\left.I_{\left(s^{(j)}=F\right)} L_{F}^{(j)}\right]$ for $i=i_{\max }$ and $L_{O}^{(i)}(j, \mathbf{s})=L_{\max }(j, \mathbf{s})$ for $i \in \mathcal{M}^{\wedge} \backslash\left\{j, i_{\max }\right\}$; and, the inspection/repair time is given by $L(j, \mathbf{s})=L_{s^{(i)}}^{(i)}(j, \mathbf{s})=\left(I_{\left(s^{(i)}=T\right)} L_{T}^{(i)}+I_{\left(s^{(i)}=F\right)} L_{F}^{(i)}\right)+I_{\left(i=i_{\max }\right)}$ $\left(I_{\left(s^{(i)}=O T\right)}\left[L_{T}^{(i)}-L_{O}^{(i)}(j, \mathbf{s})\right]^{+}+I_{\left(s^{(i)}=O F\right)}\left[L_{F}^{(i)}-L_{O}^{(i)}(j, \mathbf{s})\right]^{+}\right)$ for $i \in \mathcal{M}^{\prime}$, where $j$ is the index of the machine that triggers a system-wide stoppage.

The expected cost per operating time is given by

$$
E[T C]=\lim _{t \rightarrow \infty} \frac{\sum_{i \in \mathcal{M}^{\prime}} R_{i}(t)}{t}=\frac{\sum_{i \in \mathcal{M}} E\left[\widehat{C C}^{(i)}\right]}{E[\hat{\tau}]}
$$

where $E\left[\widehat{C C}^{(i)}\right]$ and $E[\hat{\tau}]$ denote the expected cycle cost incurred by machine $i$ and the expected operating time within a cycle, resp. $E\left[\widehat{C C}^{(i)}\right]$ consists of two components. The first corresponds to the expected cost of sampling/inspection, poor quality, and repair costs, and the latter corresponds to the expected cost of shutdown/lost profit. Each cost component 
is computed conditioned on system stoppage by a particular machine $j$ and then summed over all possible realizations of $j$ with the individual uniform occurrence probabilities of $(1 / m)$. We compute the expected operating time $E[\hat{\tau}]$ in a similar fashion. The expressions for $E\left[\widehat{C C}^{(i)}\right]$ and $E[\hat{\tau}]$ are provided in the Online Supplement.

Although the expected cost rate can be computed exactly, it may be prohibitively tedious for realistic settings. Therefore, we propose an approximation for the all-taker case such that its operating characteristics can be written in terms of the expressions for a single machine facing Poisson exogenous stoppages. This will provide us with a building block to be used later for the mixed case.

Consider the classical single machine setting $(\mu=0)$ for some machine $i$. Suppose that the total number of selfstoppages over a total $t$ of operating time can be described by a Poisson process with rate $\gamma^{(i)}$, where $\gamma^{(i)}=1 /\left.E\left[\tau^{(i)}\right]\right|_{\mu=0}$. The system stoppages per unit of operating time for a group of $m^{\prime}$ machines, all of which are opportunity takers, would also constitute a Poisson process with rate $\Gamma=\sum_{k=1}^{m^{\prime}} \gamma^{(k)}$. Then, machine $i$, taken in isolation in this group, can be modeled as a single machine facing exogenous opportunities with rate $\mu^{(i)}=\left(\Gamma-\gamma^{(i)}\right)$. This would indeed be the case if all machines were continuously monitored and no Type I or Type II errors were present $\left(h^{(i)}=0, \alpha^{(i)}=\beta^{(i)}=0 \forall i \in \mathcal{M}^{\prime}\right)$ because process shifts are exponential. But, because of nonzero sampling errors and positive sampling intervals, the actual stoppages are clearly non-Markovian. Yet, in large systems with diverse machine characteristics, the sampling intervals would be of different lengths with values over a wide range and, hence, it would be reasonable to assume that a Poisson process describes system stoppages. (We discuss the goodness of the approximation in the numerical study section below.) Building on this, we construct an approximation to $E[T C]$ as follows.

$$
\begin{aligned}
E\left[\widehat{C C^{(i)}}\right] & \approx E\left[\widetilde{C C^{(i)}}\right] \\
& =\left.\sum_{s^{(i)} \in\{T, F, O T, O F\}} E\left[C C_{s^{(i)}}^{(i)}\right]\right|_{\mu^{(i)}=\left(\Gamma-\gamma^{(i)}\right)} \\
+\pi & {\left[\sum_{j} \sum_{s^{(1)}, \ldots, s^{(m)}} L_{s^{(i)}}^{(i)}(j, \mathbf{s}) \Phi(i, j, \mathbf{s}) P_{s^{(i)}}^{(i)}\left(\Gamma-\gamma^{(i)}\right)\right] }
\end{aligned}
$$

where

$$
\begin{aligned}
\Phi(i, j, \mathbf{s})= & \prod_{k \in \mathcal{M}_{\text {TK } \backslash\{i, j\}}} \frac{I_{\left(s^{(k)}=O T\right)} P_{O T}^{(k)}\left(\Gamma-\gamma^{(k)}\right)+I_{\left(s^{(k)}=O F\right)} P_{O F}^{(k)}\left(\Gamma-\gamma^{(k)}\right)}{P_{O T}^{(k)}\left(\Gamma-\gamma^{(k)}\right)+P_{O F}^{(k)}\left(\Gamma-\gamma^{(k)}\right)} \\
& \times\left[I_{(i=j)}+\frac{\gamma^{(j)}}{\Gamma-\gamma^{(i)}} \frac{I_{(i \neq j)}\left[I_{\left(s^{(j)}=T\right)} P_{T}^{(j)}\left(\Gamma-\gamma^{(j)}\right)+I_{\left(s^{(j)}=F\right)} P_{F}^{(j)}\left(\Gamma-\gamma^{(j)}\right)\right]}{P_{T}^{(j)}\left(\Gamma-\gamma^{(j)}\right)+P_{F}^{(j)}\left(\Gamma-\gamma^{(j)}\right)}\right]
\end{aligned}
$$

denotes the probability that status of machine $i$ is $s^{(i)}$, machine $j$ in status $s^{(j)}$ has signaled a self-stoppage and machines $k\left(\in \mathcal{M}_{\mathrm{TK}} \backslash\{i\}\right)$ are found in status $s^{(k)}$ at the time of stoppage. Letting, for $i \in \mathcal{M}^{\prime}$,

$$
\bar{L}_{s^{(i)}}^{(i)}=\sum_{j} \sum_{\left.s^{(k)}: \forall k \in \mathcal{M} / \backslash i i\right\}} L_{s^{(i)}}^{(i)}(j, \mathbf{s}) \Phi(i, j, \mathbf{s})
$$

we have

$$
\begin{aligned}
& E\left[\widetilde{C C^{(i)}}\right]=\sum_{s^{(i)} \in\{T, F, O T, O F\}} \\
& \quad\left[\left.E\left[C C_{s^{(i)}}^{(i)}\right]\right|_{\mu^{(i)}=\left(\Gamma-\gamma^{(i)}\right)}+\pi \bar{L}_{s^{(i)}}^{(i)} P_{s^{(i)}}^{(i)}\left(\Gamma-\gamma^{(i)}\right)\right] .
\end{aligned}
$$

Similarly, $E\left[\widetilde{\tau^{(i)}}\right]=\sum_{s^{(i)} \in\{T, F, O T, O F\}} E\left[\left.\tau_{s^{(i)}}^{(i)}\right|_{\mu^{(i)}=\left(\Gamma-\gamma^{(i)}\right)}\right.$. Hence,

$$
E[T C] \approx E[\widetilde{T C}]=\sum_{i=1}^{m} \frac{E\left[\widetilde{C C^{(i)}}\right]}{E\left[\widetilde{\tau^{(i)}}\right]}
$$

Thus, we have an approximation to the expected cost rate for the multimachine setting in terms of individual single-machine models when all machines are opportunitytakers.

\subsection{Mixed Model}

Finally, consider the case where some of the machines are opportunity takers and some are opportunity nontakers, i.e. $\mathcal{M}_{\mathrm{NTK}} \neq \emptyset, \delta^{(i)}=0$ for $i \in \mathcal{M}_{\mathrm{NTK}}$ and, $\mathcal{M}_{\mathrm{TK}} \neq \emptyset$, $\delta^{(i)}=1$ for $i \in \mathcal{M}_{\mathrm{TK}}$. (Clearly, $\mathcal{M}=\mathcal{M}_{\mathrm{TK}} \cup \mathcal{M}_{\mathrm{NTK}}$.) Consider the following scenario. Machine $j$ signals an alarm causing a system-wide stoppage. At the system restart, each machine $i\left(\in \mathcal{M}^{\prime}\right)$ will have been restored to in-control status and the remaining time until its first sampling instance, say $\eta^{(i)}$, will be equal to $h^{(i)}$. On the other hand, for each machine $i\left(\in \mathcal{M}_{\mathrm{NTK}} \backslash\{j\}\right)$, the true shift status will not be known and $0<\eta^{(i)}<h^{(i)}$. Theoretically, one could model the system via an embedded continuous valued Markov chain where the system state is defined by the shift status 
of each machine (binary state variable) and time until its first sampling instance (continuous state variable). However, even with discretization, the size of the state space makes this approach computationally impractical for realistic settings. Therefore, we employ the approximation approach introduced above and propose the following.

$$
\begin{aligned}
E[T C] & =\lim _{t \rightarrow \infty} \frac{\{\text { Expected cost incurred by the system up to operating time } t\}}{t} \\
& =\sum_{i \in \mathcal{M}_{\mathrm{TK}}} \lim _{t \rightarrow \infty} \frac{R_{i}(t)}{t}+\sum_{i \in \mathcal{M}_{\mathrm{NTK}}} \lim _{t \rightarrow \infty} \frac{R_{i}(t)}{t} \\
& \approx E[\widetilde{T C}] \\
& =\sum_{i \in \mathcal{M}_{\mathrm{TK}}} \frac{\left.E\left[C C^{(i)}\right]\right|_{\mu^{(i)}=\Gamma-\gamma^{(i)}}}{\left.E\left[\tau^{(i)}\right]\right|_{\mu^{(i)}=\Gamma-\gamma^{(i)}}}+\sum_{i \in \mathcal{M}_{\mathrm{NTK}}} \frac{\left.E\left[C C^{(i)}\right]\right|_{\mu^{(i)}=0}}{\left.E\left[\tau^{(i)}\right]\right|_{\mu^{(i)}=0}} \\
& =\sum_{i \in \mathcal{M}}\left[\delta^{(i)} \frac{\left.E\left[C C^{(i)}\right]\right|_{\mu^{(i)}=\Gamma-\gamma^{(i)}}}{\left.E\left[\tau^{(i)}\right]\right|_{\mu^{(i)}=\Gamma-\gamma^{(i)}}}+\left(1-\delta^{(i)}\right) \frac{\left.E\left[C C^{(i)}\right]\right|_{\mu^{(i)}=0}}{\left.E\left[\tau^{(i)}\right]\right|_{\mu^{(i)}=0}}\right]
\end{aligned}
$$

where $\Gamma=\sum_{k=1}^{m} \gamma^{(k)}$ as before. We address the goodness of this approximation in our numerical study.

\section{MULTIPLE MACHINES: NUMERICAL STUDY}

We have conducted a numerical study for the multiple machine setting to investigate (i) the opportunity taking behavior of a group of machines operating under jidoka, (ii) the goodness of the proposed approximation, and (iii) the cost advantages of employing the models herein versus the classical model where opportunities are not taken into account. For our numerical study, we consider an $\bar{X}$ control chart with $X$ as defined above.

For the experimental set, we set $m=8, b=0.1$, $u=5, R_{T}=R_{F}=0$, and $L_{T}=L_{F}=L$. We varied the rest of the parameters as follows: $\pi \in\{500,1500\}$, $\lambda \in\{0.01,0.03,0.05,0.06,0.07,0.08,0.09,0.1\}, L \in$ $\{0.025,0.05,0.075,0.1,0.125,0.15,0.2,0.25\}$ and $a \in$ $\{50,150,250,300,350,400,450,500\}$. Thus, for each value of $\pi$, we consider 14 different experiments depending on the values of $\lambda, a$, and $L$ as summarized in Table 4 , where $(\leftrightarrow)$ indicates that the corresponding parameter is identical for all of the machines, ( $\nearrow$ ) indicates that values of the corresponding parameter assigned to the machines from lowest to highest (e.g., $\lambda^{(1)}=0.01$ and $\lambda^{(8)}=0.1$ ), and, $\searrow$ ) indicates the opposite assignment from highest to lowest. For each experiment, we determined (i) the best control policy parameter values and (ii) the best partitioning of machines, which jointly yield the lowest expected cost rate obtained from the analytical models. The cost rates were computed exactly through Eq. (10) for the cases where all of the machines were opportunity nontakers, and approximately via Eqs. (16) and (17) for the cases where all or some of the machines were opportunity takers. The joint optimization of these models was done iteratively with the use of three algorithms as described below [25].

In the multiple machine setting, determination of the optimal policy parameter triplet $\left(y^{*(i)}, k^{*(i)}, h^{*(i)}\right) \forall i \in M$ requires joint optimization for $m$ machines, since the opportunity rates $\mu^{(i)}$ depend on the policy parameters of the other machines. We do this via a convergence algorithm employing the optimization algorithm in Section 4. For each given partitioning of the machines, we (i) begin with finding the optimal policy parameter triplet for no opportunity arrivals; (ii) compute the corresponding opportunity rates that each machine would experience when the machines are operating under these policy parameter values; (iii) find the new optimal policy parameter triplets for each facing these opportunity arrival rates; (iv) repeat this until we obtain sufficient convergence in the expected cost rate in two successive iterations. Although cost convergence was achieved for all of the experiments in our numerical study, we cannot guarantee convergence. Finding the optimal partitioning of $m$ machines into sets $M_{\mathrm{TK}}$ and $M_{\text {NTK }}$ requires searching over all $2^{m}$ possible machine partitioning. Therefore, we have, instead, employed a onepass greedy heuristic for separating the machines on the

Table 4. Experimental set for the multiple machine numerical study.

\begin{tabular}{cccccccc}
\hline $\operatorname{Exp} \#$ & $\lambda$ & $L$ & $a$ & $\operatorname{Exp} \#$ & $\lambda$ & $L$ & $a$ \\
\hline 1 & $\leftrightarrow$ & $\leftrightarrow$ & $\leftrightarrow$ & 8 & $\nearrow$ & $\leftrightarrow$ & $\searrow$ \\
2 & $\searrow$ & $\leftrightarrow$ & $\leftrightarrow$ & 9 & $\leftrightarrow$ & $\nearrow$ & $\nearrow$ \\
3 & $\leftrightarrow$ & $\nearrow$ & $\leftrightarrow$ & 10 & $\leftrightarrow$ & $\nearrow$ & $\searrow$ \\
4 & $\leftrightarrow$ & $\leftrightarrow$ & $\nearrow$ & 11 & $\nearrow$ & $\nearrow$ & $\nearrow$ \\
5 & $\nearrow$ & $\nearrow$ & $\leftrightarrow$ & 12 & $\searrow$ & $\nearrow$ & $\nearrow$ \\
6 & $\searrow$ & $\nearrow$ & $\leftrightarrow$ & 13 & $\searrow$ & $\nearrow$ & $\searrow$ \\
7 & $\nearrow$ & $\leftrightarrow$ & $\nearrow$ & 14 & $\nearrow$ & $\nearrow$ & $\searrow$ \\
\hline
\end{tabular}


Table 5. Partitioning of machines into $M_{\mathrm{NTK}}$ and $M_{\mathrm{TK}}$.

\begin{tabular}{|c|c|c|c|c|c|c|c|c|c|c|c|c|c|c|c|c|}
\hline \multirow[b]{3}{*}{ Exp \# } & \multicolumn{8}{|c|}{$\pi=500$} & \multicolumn{8}{|c|}{$\pi=1500$} \\
\hline & \multicolumn{8}{|c|}{ Machine \# } & \multicolumn{8}{|c|}{ Machine \# } \\
\hline & 1 & 2 & 3 & 4 & 5 & 6 & 7 & 8 & 1 & 2 & 3 & 4 & 5 & 6 & 7 & 8 \\
\hline 1 & $T$ & $T$ & $T$ & $T$ & $T$ & $T$ & $T$ & $T$ & $T$ & $T$ & $T$ & $T$ & $T$ & $T$ & $T$ & $T$ \\
\hline 2 & $T$ & $T$ & $T$ & $T$ & $T$ & $T$ & $T$ & $T$ & $T$ & $T$ & $T$ & $T$ & $T$ & $T$ & $T$ & $T$ \\
\hline 3 & $T$ & $T$ & $T$ & $\mathbf{N}$ & $\mathbf{N}$ & $\mathbf{N}$ & $\mathbf{N}$ & $\mathbf{N}$ & $T$ & $T$ & $\mathbf{N}$ & $\mathbf{N}$ & $\mathbf{N}$ & $\mathbf{N}$ & $\mathbf{N}$ & $\mathbf{N}$ \\
\hline 4 & $T$ & $T$ & $T$ & $T$ & $T$ & $T$ & $T$ & $T$ & $T$ & $T$ & $T$ & $T$ & $T$ & $T$ & $T$ & $T$ \\
\hline 5 & $T$ & $T$ & $T$ & $T$ & $T$ & $\mathbf{N}$ & $\mathbf{N}$ & $\mathbf{N}$ & $T$ & $T$ & $T$ & $T$ & $\mathbf{N}$ & $\mathbf{N}$ & $\mathbf{N}$ & $\mathbf{N}$ \\
\hline 6 & $T$ & $T$ & $\mathbf{N}$ & $\mathbf{N}$ & $\mathbf{N}$ & $\mathbf{N}$ & $\mathbf{N}$ & $\mathbf{N}$ & $\mathbf{N}$ & $\mathbf{N}$ & $\mathbf{N}$ & $\mathbf{N}$ & $\mathbf{N}$ & $\mathbf{N}$ & $\mathbf{N}$ & $\mathbf{N}$ \\
\hline 7 & $T$ & $T$ & $T$ & $T$ & $T$ & $T$ & $T$ & $T$ & $T$ & $T$ & $T$ & $T$ & $T$ & $T$ & $T$ & $T$ \\
\hline 8 & $T$ & $T$ & $T$ & $T$ & $T$ & $T$ & $T$ & $T$ & $T$ & $T$ & $T$ & $T$ & $T$ & $T$ & $T$ & $T$ \\
\hline 9 & $T$ & $T$ & $T$ & $\mathbf{N}$ & $\mathbf{N}$ & $\mathbf{N}$ & $\mathbf{N}$ & $\mathbf{N}$ & $T$ & $T$ & $\mathbf{N}$ & $\mathbf{N}$ & $\mathbf{N}$ & $\mathbf{N}$ & $\mathbf{N}$ & $\mathbf{N}$ \\
\hline 10 & $T$ & $T$ & $T$ & $\mathbf{N}$ & $\mathbf{N}$ & $\mathbf{N}$ & $\mathbf{N}$ & $\mathbf{N}$ & $T$ & $\mathbf{N}$ & $\mathbf{N}$ & $\mathbf{N}$ & $\mathbf{N}$ & $\mathbf{N}$ & $\mathbf{N}$ & $\mathbf{N}$ \\
\hline 11 & $T$ & $T$ & $T$ & $T$ & $T$ & $\mathbf{N}$ & $\mathbf{N}$ & $\mathbf{N}$ & $T$ & $T$ & $T$ & $T$ & $\mathbf{N}$ & $\mathbf{N}$ & $\mathbf{N}$ & $\mathbf{N}$ \\
\hline 12 & $T$ & $T$ & $\mathbf{N}$ & $\mathbf{N}$ & $\mathbf{N}$ & $\mathbf{N}$ & $\mathbf{N}$ & $\mathbf{N}$ & $T$ & $\mathbf{N}$ & $\mathbf{N}$ & $\mathbf{N}$ & $\mathbf{N}$ & $\mathbf{N}$ & $\mathbf{N}$ & $\mathbf{N}$ \\
\hline 13 & $T$ & $T$ & $\mathbf{N}$ & $\mathbf{N}$ & $\mathbf{N}$ & $\mathbf{N}$ & $\mathbf{N}$ & $\mathbf{N}$ & $T$ & $\mathbf{N}$ & $\mathbf{N}$ & $\mathbf{N}$ & $\mathbf{N}$ & $\mathbf{N}$ & $\mathbf{N}$ & $\mathbf{N}$ \\
\hline 14 & $T$ & $T$ & $T$ & $T$ & $T$ & $\mathbf{N}$ & $\mathbf{N}$ & $\mathbf{N}$ & $\mathbf{N}$ & $\mathbf{N}$ & $\mathbf{N}$ & $\mathbf{N}$ & $\mathbf{N}$ & $\mathbf{N}$ & $\mathbf{N}$ & $\mathbf{N}$ \\
\hline
\end{tabular}

basis of the expected cost rate differential; we begin with $\mathcal{M}_{\mathrm{NTK}}=\emptyset$ and assign machines one by one to $\mathcal{M}_{\mathrm{NTK}}$ on the basis of the differential obtained in the expected cost rate. The heuristic requires considering at most $m(m+1) / 2$ partitions. The results were also tested via simulation.

\subsection{Opportunity-Taker Partitioning}

We begin the discussion of our findings with the results on partitioning of the machines into the opportunity taker and opportunity nontaker sets. The partitionings address the issue of whether or not it is beneficial for a machine to utilize the opportunities in a particular setting. In the single machine setting, we observed that only the opportunities with $L_{O F}=0\left(=\left[L_{F}-L_{O}\right]^{+}\right)$are beneficial for reducing the cost per operating time. Thus, a simple rule for opportunity taking behavior would be to take only those opportunities that qualify in this manner, if the durations of opportunities were known with certainty. However, in the multiple machine setting, although one knows which machine has signaled an alarm triggering a system-wide stoppage, the shift status of this machine and of all the other opportunity taking machines are unknown. Hence, the duration of an opportunity is effectively a random variable; and, a simple deterministic rule as such cannot guarantee an optimal partitioning.

The partitionings that we obtained for the experimental set are shown in Table 5 where $T$ denotes that a machine is an opportunity taker machine and $\mathbf{N}$ denotes that a machine is an opportunity nontaker machine. We see that, only in one experiment, we have $M_{\mathrm{TK}}=\emptyset$; in the rest, there is at least one opportunity taker machine. In 10 out of 28 experiments, all of the machines are opportunity takers. Partitioning is observed to be primarily determined by parameter $L$, and the machines with smaller $L$ tend to be opportunity takers (e.g., compare
Experiments \#7,\#11, and \#13.) This is to be expected, since the machines with larger inspection/repair times would result in longer idle times and higher lost opportunity costs if they were opportunity takers. This is also consistent with the single machine results. The parameters $\lambda$ and $a$ have almost no or little effect on the partitioning by themselves but $\lambda$ has a confounding effect on $L$ (e.g., compare Experiments \#3, \#9, and \#10.)

\subsection{Goodness of the Approximation}

As the benchmark, we use the expected cost rates obtained by simulating the system under the given partitioning and the corresponding "optimal" policy parameter values determined analytically. For each experiment, we considered the cases: (i) all machines are opportunity nontakers, (ii) all machines are opportunity takers and, (iii) the optimal partition is achieved. Table 6 tabulates the expected cost rate computed analytically and the percentage deviation from that obtained via simulation, $\%$ Err $=100 \times\left(E\left[T C_{\text {simulation }}\right]-\right.$ $\left.E\left[T C_{\text {analytical }}\right]\right) / E\left[T C_{\text {simulation }}\right]$. The analytically computed cost rate for the all nontaker case is exact; therefore, the observed error in this case is an indicator of simulation errors. We see that the simulation error is quite small. For $\pi=500$, the mean is $-0.07 \%$, and the median is $-0.03 \%$; for $\pi=1500$, the statistics are $0.27 \%$ and $0.35 \%$. Overall, the maximum absolute deviation is less than $0.8 \%$. For the all-taker and mixed cases, the analytical model implies the approximate model developed above. Hence, the percentage deviations between the analytical and simulation solutions for these cases indicate the performance of the proposed approximation (in addition to the simulation errors). Our simulation results show that there is no consistent over- or underestimation arising from the proposed approximation. We observe 
Table 6. Analytical cost rate, $E\left[T C_{\text {analytical }}\right]$ and deviation from simulation, \%Err.

\begin{tabular}{|c|c|c|c|c|c|c|}
\hline \multirow[b]{2}{*}{ Exp \# } & \multicolumn{3}{|c|}{$\pi=500$} & \multicolumn{3}{|c|}{$\pi=1500$} \\
\hline & All Nontaker & All taker & Partitioned & All Nontaker & All taker & Partitioned \\
\hline 1 & $126.26 ;-0.10$ & $110.22 ; 8.19$ & $110.22 ; 8.19$ & $185.48 ; 0.77$ & $157.9 ; 5.95$ & $157.9 ; 5.95$ \\
\hline 2 & $206.86 ;-0.03$ & $185.55 ; 1.22$ & $185.55 ; 1.22$ & $327.75 ; 0.30$ & $288.66 ; 0.83$ & $288.66 ; 0.83$ \\
\hline 3 & $120.51 ;-0.43$ & $131.44 ;-0.55$ & $117.81 ; 0.04$ & $168.75 ; 0.34$ & $219.90 ;-4.96$ & $167.03 ; 0.59$ \\
\hline 4 & $132.59 ; 0.03$ & $116.06 ; 1.67$ & $116.06 ; 1.67$ & $191.72 ; 0.45$ & $160.43 ; 2.67$ & $160.43 ; 2.67$ \\
\hline 5 & $139.47 ; 0.32$ & $144.11 ;-2.58$ & $132.95 ;-0.82$ & $213.00 ; 0.44$ & $248.41 ;-4.69$ & $207.73 ;-0.03$ \\
\hline 6 & $123.96 ;-0.10$ & $145.85 ;-3.04$ & $121.72 ; 0.63$ & $167.05 ; 0.30$ & $254.50 ;-7.10$ & $167.05 ; 0.30$ \\
\hline 7 & $141.22 ; 0.28$ & $121.43 ; 1.98$ & $121.43 ; 1.98$ & $213.40 ; 0.56$ & $176.74 ; 2.03$ & $176.74 ; 2.03$ \\
\hline 8 & $123.65 ; 0.21$ & $102.12 ; 3.04$ & $102.12 ; 3.04$ & $194.27 ; 0.22$ & $148.76 ; 6.45$ & $148.76 ; 6.45$ \\
\hline 9 & $114.86 ; 0.27$ & $125.42 ;-4.11$ & $111.79 ;-0.43$ & $163.10 ; 0.59$ & $210.75 ;-9.72$ & $160.47 ;-1.01$ \\
\hline 10 & $114.26 ;-0.61$ & $122.79 ;-0.84$ & $111.77 ; 0.13$ & $161.77 ; 0.36$ & $197.49 ;-0.04$ & $160.24 ;-0.57$ \\
\hline 11 & $142.08 ; 0.19$ & $147.27 ;-2.62$ & $135.38 ;-0.32$ & $216.01 ;-0.10$ & $253.87 ;-6.69$ & $209.51 ;-1.22$ \\
\hline 12 & $109.37 ;-0.72$ & $129.71 ;-4.99$ & $106.75 ; 0.48$ & $152.11 ; 0.60$ & $224.91 ;-11.72$ & $149.48 ; 0.10$ \\
\hline 13 & $139.47 ;-0.60$ & $161.94 ;-2.23$ & $137.35 ;-0.10$ & $182.76 ;-0.40$ & $270.10 ;-4.69$ & $180.91 ;-0.06$ \\
\hline 14 & $123.86 ;-0.19$ & $124.08 ;-1.45$ & $117.68 ;-0.89$ & $194.77 ;-0.05$ & $200.86 ;-1.76$ & $194.77 ;-0.05$ \\
\hline
\end{tabular}

that the approximation has the worst performance for the case of identical machines (Experiment \#1). In this case, the machines are sampled in locked step and the time between system stoppages is given by the minimum of iid geometric variables. Clearly, the Poisson assumption of opportunity arrivals is least applicable here. The approximation performance gets better as the sampling intervals start differing from each other, which happens as the idleness cost and/or the diversity of machine characteristics and/or the number of opportunity nontakers increase. To see this, we compare Experiments \#1 and \#2 for $\pi=500$ and 1500. For Experiment $\# 1$ and $\pi=500$, we see the largest percentage error $8.19 \%$. In this instance, all machines are identical, they are all opportunity takers and the optimal sampling intervals are $h^{*(1)}=1.106$ and $h^{*(i)}=1.01$ for $i=2, \ldots, 8$. However, for Experiment \#2 where the machines differ in their reliabilities, the optimal partitioning is again all opportunity takers but we have the optimal sampling intervals as $\mathbf{h}^{*}=$ $\{0.538,0.572,0.608,0.653,0.708,0.779,1.024,1.891\}$; the diversity in the sampling intervals reduces the error to $1.22 \%$. For $\pi=1500$, we have $h^{*(1)}=1.308, h^{*(i)}=1.329$ for $i=$ $2, \ldots, 8$ for Experiment \#1 and $\mathbf{h}^{*}=\{0.638,0.684,0.728$, $0.779,0.847,0.933,1.230,2.295\}$ for Experiment \#2; the respective errors are $5.95 \%$ and $0.83 \%$. In all four instances, $y^{*}$ and $k^{*}$ values are almost identical for all machines, leaving the sampling interval as the differentiating factor. We can conclude that the Poisson assumption of opportunity arrivals is a good approximation for diverse systems where sampling intervals vary across machines.

\subsection{Advantages of JPC}

Finally, we consider the cost advantages achieved by the introduction of JPC instead of using the classical setting where all machines are opportunity nontakers.
We present the percentage improvements in Table 7 where each entry is $\% \Delta=100 \times\left[\left.E[T C]\right|_{\mathrm{PAll} \_\mathrm{NTK}}-\right.$

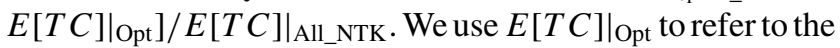
optimal expected cost per operating time achieved under JPC, and $\left.E[T C]\right|_{\text {All_NTK }}$ corresponds to the classical model. We calculated the percentage improvements with both the analytically computed cost rates and the simulated results. To obtain the simulated optimal cost rate, we first determined the optimal control policy parameter values and partitionings via the models developed herein and, then, simulated the system in this optimal setting. To obtain the simulated all nontaker cost rate, we simulated the case of all nontaker machines with their

Table 7. Percentage improvement, $\% \Delta$, and the summary statistics in the multiple machine model.

\begin{tabular}{ccc}
\hline & $\pi=500$ & $\pi=1500$ \\
\cline { 2 - 3 } Exp \# & $\% \Delta$ & $\% \Delta$ \\
\hline 1 & 4.82 & 10.18 \\
2 & 9.16 & 11.48 \\
3 & 1.86 & 0.78 \\
4 & 11.00 & 14.42 \\
5 & 5.75 & 2.93 \\
6 & 1.09 & 0 \\
7 & 12.53 & 15.95 \\
8 & 14.98 & 18.32 \\
9 & 3.34 & 3.18 \\
10 & 1.46 & 1.86 \\
11 & 5.20 & 4.08 \\
12 & 1.22 & 2.22 \\
13 & 1.03 & 0.68 \\
14 & 5.66 & 0 \\
Mean & 5.65 & 6.15 \\
Median & 5.01 & 3.05 \\
Min & 1.03 & 0 \\
Max & 14.98 & 18.32 \\
\hline
\end{tabular}


optimal policy parameter values determined via Eq. (10). We observed that the deviations between the analytically computed and simulated cost rates are consistent with the results reported above on the goodness of the approximation. We also observed that the qualitative findings on improvements are the same for both analytically computed and simulated cost rates. In Table 7, we report the simulated results. The percentage improvements provided by JPC are between $1.03 \%$ and $14.98 \%$ with the mean and median being $5.65 \%$ and $5.01 \%$ for $\pi=500$ and, between $0 \%$ and $18.32 \%$ with the mean and median being $6.15 \%$ and $3.05 \%$ for $\pi=1500$. As expected, maximum improvements occur when all machines are opportunity takers. We conclude that JPC can provide significant savings in multiple machine settings, as well.

\section{CONCLUSION}

In this article, we consider design of control charts in the presence of machine stoppages that are exogenously imposed. Each stoppage creates an opportunity for inspection/repair at reduced cost. We first model a single machine facing opportunities arriving according to a Poisson process, develop the expressions for its operating characteristics and construct the optimization problem for economic design of a control chart. We, then, consider a multiple machine setting where alarms about the quality status of the machines cause system-wide stoppages as it is the case under jidoka practices. We develop exact expressions for the cases where all of the machines are either opportunity-takers or nontakers, and propose an approximate model for the mixed case. In a numerical study, we examine the opportunity taking behavior of machines in both single and multiple machine settings and the impact of such practices on the design of an $\bar{X}$ control chart. Our findings indicate that ignoring exogenous inspection/repair opportunities and employing the classical QC chart parameters may result in significant cost increases.

There are a number of extensions to our basic model. Herein, we consider only the design of $\bar{X}$ control charts in our numerical study, but our model in the presence of opportunistic inspections can be applied to other variableand attribute-control charts. Similarly, different design criteria (semieconomic and statistical) can be considered, as well. Furthermore, opportunity arrivals may be generalized to non-Markovian processes.

\section{REFERENCES}

[1] M.S. Bazaraa, H.D. Sherali, and C.M. Shetty, Nonlinear programming, John Wiley \& Sons, Inc. USA, 1993.
[2] W.K. Chiu, Economic design of np charts for processes subject to a multiplicity of assignable causes, Management Sci 23 (1976), 404-411.

[3] W.K. Chiu, Minimum cost control schemes using np charts, Int J Prod Res 13 (1975), 341-349.

[4] W.K. Chiu, Economic design of attribute control charts, Technometrics 17 (1975), 81-87.

[5] D.J. Davis, An analysis of some failure data, J Am Stat Assoc 47 (1952), 113-150.

[6] R. Dekker and M.C. Dijkstra, Opportunity-based age replacement: Exponentially distributed times between opportunities, Nav Res Logist 39 (1992), 175-190.

[7] A.J. Duncan, The economic design of $X$ charts used to maintain current control of process, J Am Stat Assoc 51 (1956), 228-242.

[8] B. Epstein, The exponential distribution and its role in lifetesting, Ind Qual Control 15 (1958), 2-7.

[9] I.N. Gibra, Economically optimal determination of the parameters of $\bar{X}$-control chart, Management Sci 17 (1971), 635-646.

[10] I.N. Gibra, Recent developments in control chart techniques, J Qual Technol 7 (1975), 183-192.

[11] A.L. Goel, S.C. Jain, and S.M. Wu, An algorithm for the determination of the economic design of $\bar{X}$-charts based on duncan's model, J Am Stat Assoc 63 (1968), 304-320.

[12] A.L. Goel and S.M. Wu, Economically optimum design of cusum charts, Management Sci 19 (1973), 1271-1282.

[13] C. Ho and K.E. Case, Economic design of control charts: A literature review for 1981-1991, J Qual Technol 26 (1994), $39-53$.

[14] E. Jahovic, H. Basic, M. Pasic, and I. Bijelonya, "Kaizen optimization modeling of tube manufacturing processes in Mercedes-Benz Turk," In: 9th International Research/Expert Conference, Antalya, Turkey, 2005.

[15] J.B. Keats, J.D. Miskulin, and G.C. Runger, Statistical process control scheme design, J Qua Technol 27 (1995), 214-225.

[16] J.L. Lorenzen and L.C. Vance, The economic design of control chart: A unified approach, Technometrics 28 (1986), 3-10.

[17] D.C. Montgomery, The economic design of control charts: A review and literature survey, J Qual Technol 12 (1980), 75-87.

[18] D.C. Montgomery, Economic design of an $\bar{X}$ control chart, J Qual Technol 14 (1982), 40-43.

[19] E.S. Page, Continuous inspection schemes, Biometrika 41 (1954), 100-114.

[20] T. Pardi, Redefining the Toyota Production System: The European side of the story, New Techonol Work Employ 22 (2007), $2-20$.

[21] S.M. Ross, Introduction to probability models, Academic Press, San Diego, CA, 1993.

[22] E.M. Saniga, Joint economically optimal design of $\bar{X}$ and $R$ control charts, Management Sci 24 (1977), 420-431.

[23] G. Tagaras, A survey of recent developments in the design of adaptive control charts, J Qual Technol 30 (1998), 212-231.

[24] Toyota Motor Corporation, Public Affairs Division Report: The Thinking Production System, 2003.

[25] A.O. Toy, Quality and production control with opportunities and exogenous random shocks, Bilkent University, 2005.

[26] L.C. Vance, A bibliography of statistical quality control chart techniques, 1970-1980, J Qual Technol 15 (1983), 59-62. 